\title{
High Thoracic Epidural Anaesthesia for Endocardial Ablation of Atrial Fibrillation: A Single Centre Experience
}

\author{
Angela Lappa MD ${ }^{1}$, Marzia Cottini MD ${ }^{2 *}$, Patrizia Picozzi MD ${ }^{1}$, Silvia Donfrancesco MD $^{1}$, \\ Davino Emilio MD ${ }^{1}$, Valentina Coltelli MD ${ }^{1}$, Di Pirro Lucio MD $^{1}$, , Luca Severi MD ${ }^{1}$, Francesco \\ Musumeci Prof, MD $^{2}$ \\ ${ }^{I}$ Department of Anaesthesia, Intensive Care and Pain therapy, Cardiovascular Intensive Care Unit, "S. \\ Camillo- Forlanini” Hospital, 00152 Rome, Italy \\ ${ }^{2}$ Department of Heart and Vessels, Cardiac Surgery Unit and Heart Transplantation Center, "S. Camillo- \\ Forlanini” Hospital, 00152 Rome, Italy
}

\begin{abstract}
"Corresponding Author: Marzia Cottini, Department of Heart and Vessels, Cardiac Surgery Unit and Heart Transplantation Center, "S. Camillo-Forlanini" Hospital, 00152 Rome, Italy, PhD Student, University La Sapienza of Rome E-mail: marzia.cottini @gmail.com
\end{abstract}

\begin{abstract}
Catheter ablation could be an optimal therapy for permanent atrial fibrillation. We investigate the best anesthesiological management to improve this procedure. We collected the data of 20 consecutive patients undergone to catheter ablation due to permanent/paroxysmal atrial fibrillation with similar clinical and pathological history. According to our experience the use of high thoracic epidural anaesthesia (HTEA) for endocardial ablation of $A F$ was an optimal, reliable and satisfactory. In expertise anesthesiological team, it could be reduce and control immediately the neurological complications and peri-postprocedural pain because the patient was awake. Also, in patient with obesity and clinical history of respiratory disease, it could help to avoid general anaesthesia and the possible complication of intubation and long mechanical ventilatory supports.
\end{abstract}

Keywords: catheter ablation, atrial fibrillation, sedation, epidural anaesthesia.

\section{INTRODUCTION}

Atrial Fibrillation (AF) is the most common arrhythmia seen in general population ${ }^{1,2}$. It has already shown to be an independent predictor of stroke and death, contributing to an estimated $15 / 20 \%$ of ischemic cerebrovascular events annually and almost doubling the risk of death compared to people with
Sinus Rhythm $(\mathrm{SR})^{3-6}$. Although the variable and multiple first-line medical treatment, antiarrhythmic drugs are either often ineffective and they are associated with serious adverse effects also. In this context, catheter ablation for AF could be a viable alternative for restoring SR (Figure 1).

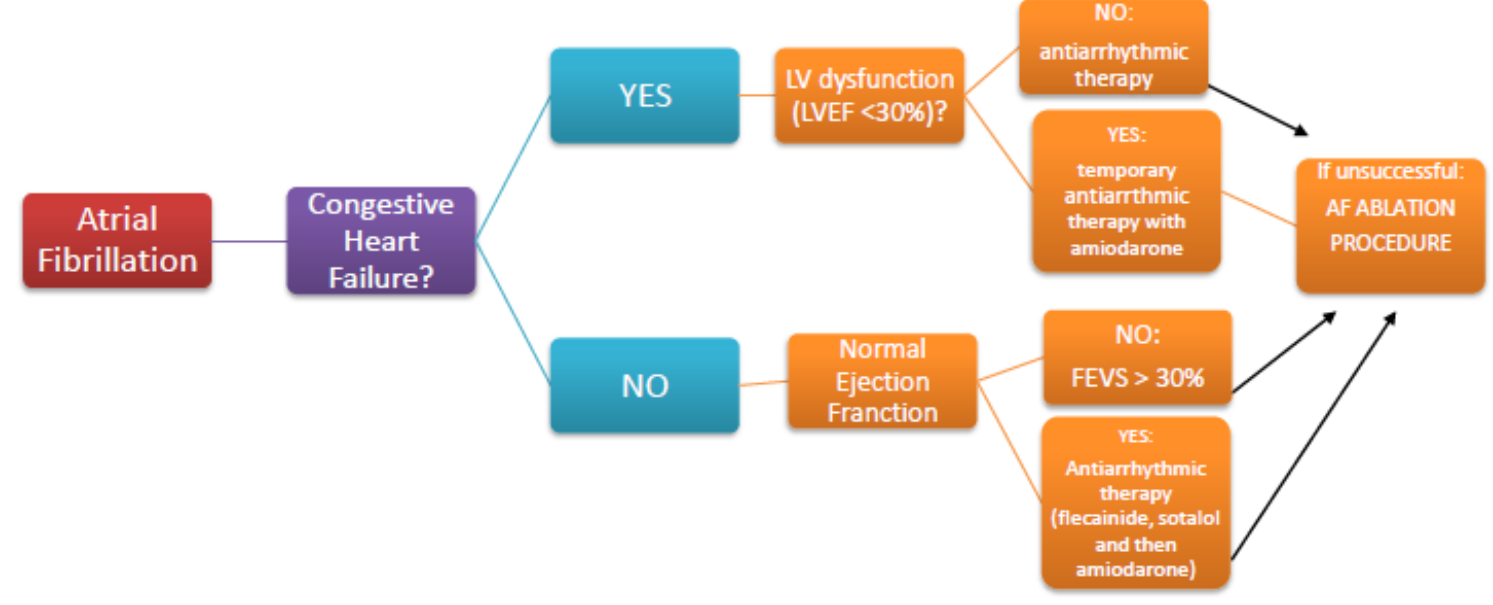

Figure1. A schematic image of AF ablation procedure indications 
The ideal candidates are patients suffering from recurrent paroxysmal $\mathrm{AF}$ without any structural heart disease. Ablative strategies have changed over time, available data are encouraging (success rate about $70 \%$ between the 1 st and 7 th year of follow up). However, more than one ablation procedure needs to control AF and the same area has to be re-ablated frequently.

Endocardial catheter ablation has two therapeutic goals: to remove all potential triggers and to stop the altered circuits of conduction that could perpetuate AF. It is performed with large series of radiofrequency pulses in the posterior atrial wall and in the antrum of the pulmonary veins, and it requires long procedural time in the most of persistent and chronic cases ${ }^{7}$. The anesthesiological aspects of that procedure have been poorly investigated. The anesthesiological goal is to provide for sedated, analgesic, amnesic, and motionless patient, maintaining stable hemodynamic conditions. Besides the patient should be cooperative with the operator to limit body movements in order to decrease difficulties to the operator and procedure duration. The anesthesiological choice should avoid respiratory depression, nausea, vomiting and should consider the control of postoperative pain. Our study investigates the feasibility, efficacy and safety of high thoracic epidural anaesthesia (HTEA) for endocardial ablation of AF.

\section{METHODS}

We had considered all symptomatic patients with unsuccessful current cardioversion and pharmacological treatments both. We enrolled 20 selected patients with coexisting respiratory pathologies and/ or obesity undergoing HTEA (Table 1).

Table1. Baseline characteristics of the patients. AF: atrial fibrillation; COPD: chronic obstructive pulmonary disease; LVEF: left ventricular ejection fraction

\begin{tabular}{|l|l|}
\hline \multicolumn{1}{|c|}{ Variable } & \multicolumn{1}{|c|}{ Value } \\
\hline $\begin{array}{l}\text { General Characteristics: } \\
\text { - Age }\end{array}$ & $55 \pm 9$ years old \\
- Gender & $\mathrm{M}: \mathrm{F}=1.2: 1$, \\
Men $\quad$ Body Mass Index & Female 45\% \\
\hline $\begin{array}{l}\text { AF history } \\
\text { - Antiarrhythmic therapy } \\
\text { - DC cardioversion }\end{array}$ & $100 \%(\mathrm{n}=20)$ \\
\hline $\begin{array}{l}\text { Cardiac pathologies } \\
\text { - AF alone }\end{array}$ & $100 \%(\mathrm{n}=20)$ \\
\hline
\end{tabular}

\begin{tabular}{|ll|l|}
\hline - & Permanent/ & $1.85: 1$ \\
& Paroxysmal AF & \\
- & Hypertension & $40 \%(\mathrm{n}=8)$ \\
- & Cardiomiopathy & $15 \%(\mathrm{n}=3)$ \\
- & Congenital Heart disease & $5 \%(\mathrm{n}=1)$ \\
- & Valvular disease & $15 \%(\mathrm{n}=3)$ \\
\hline $\begin{array}{l}\text { Respiratory pathologies } \\
\text { - Sleep apnoea syndrome }\end{array}$ & $60 \%(\mathrm{n}=12)$ \\
- COPD & $15 \%(\mathrm{n}=3)$ \\
- Asthma & $25 \%(\mathrm{n}=5)$ \\
\hline $\begin{array}{l}\text { Echocardiographic values } \\
\text { - LVEF }\end{array}$ & $40 \pm 5 \%$ \\
\hline
\end{tabular}

All patients gave written informed consent for the procedure and had been taking warfarin for at least six weeks. The last dose was taken six days before the ablation procedure and enoxaparin (1 $\mathrm{mg} / \mathrm{Kg}$ twice daily) had administered for five days before admission. A Transesophageal Echocardiogram (TEE) was performed within 24 hours of the procedure to exclude thrombus in left atrium and appendage.

Once establishing the International Number Ratio (INR) was in the range, the epidural anaesthesia was performed: medial approach and hanging drop method were employed for epidural space detection (Tuohy needle $18 \mathrm{G}$, Th 3 Level) and catheter placement. After that, an heparin bolus was given at least 60 minutes according to the American Society of Regional Anaesthesia (ASRA) ${ }^{8}$.

During ablation procedure, initial intravenous heparin bolus (100 UI/Kg) was administered and Activated Clotting Time (ACT) checked at 10 minutes and every 60 minutes subsequently. It was provided a continuous monitoring of heat rhythm, beat rate. pulse oximetry, continuous carbon dioxide measurements, invasive arterial pressure, diuresis and temperature.

Pain control was assessed by answering patients about the level of achieved analgesia and using VAS pain score (10-points visual analogue scale) with considering a good analgesia the score less than 4 (Figure 2).

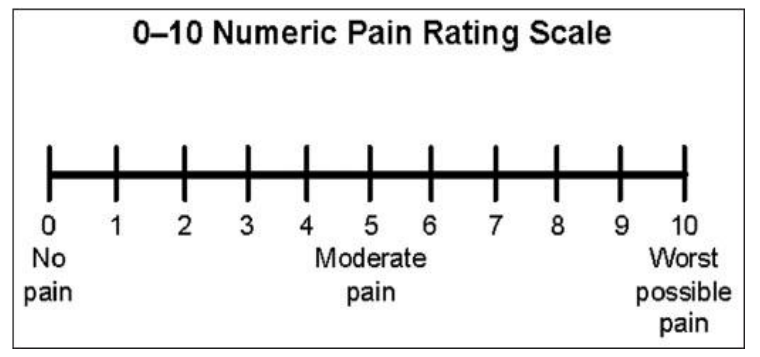

Figure2. VAS score 
At the beginning of radiofrequency ablation phase, the analgesia was achieved and maintained by a solution of $8 \mathrm{ml}$ containing ropivacaine $(38 \mathrm{mg})$ and sufentanil $(100 \mathrm{mcg})$ or fentanyl $(50 \mathrm{mcg})$.

The epidural catheter and femoral sheaths were removed after INR and PTT controls.

Ordinarily, the patients were discharged two days after and they were reloaded with warfarin and continued self enoxaparin administration until their INR achieved in therapeutic values.

\section{RESUltS}

The HTEA procedures succeeded in all cases, excepted 2 patients. Hemodynamic conditions were kept stable in each patient (medium systolic arterial pressure $110 \pm 47 \mathrm{mmHg}$, medium diastolic arterial pressure $80 \pm 11$ $\mathrm{mmHg}$ ), no prolonged hypotension or hypertension peaks were observed, pulse oximetry didn't record oxygenation desaturation, arterial carbon dioxide tension $(\mathrm{PaCO} 2)$ did not show any increase. In many patients, furosemide (10-20 mg i.v.) was required to control fluid overload during procedures and in 7 patients it was needed midazolam bolus $(2-4 \mathrm{mg})$ to improve their comfort.

According to the patients ' interviews, they referred good analgesia and mild comfort (VAS $2.7 \pm 1.3$ ), the patients' motionless was efficient and satisfying for the ablation operator.

The procedure were interrupted because of intraoperative complications:

1. The patient 1 showed a transient ischemic attack, with loss of conscience, leaf side weakness. The procedure was immediately stopped and anesthesiological and medical support were supplied to stabilize the clinical parameters. The following brain CT excluded hemorrhagic stroke. The patient had complete regression after 7 days of neurological deficits.

2. The procedure of patient 2 was complicated by cardiac tamponade, and he had immediately undergone to pericardio centesis.

Both patients was discharged after an observational period in the ward and with stable neurological or hemodynamic conditions.

\section{DISCUSSION}

Endocardial ablation of AF has improved the quality of life for many patients with untreatable supraventricular arrhythmia. In addition, it provides a significant improvement in left ventricle (LV) performance and quality of life in patients with $\mathrm{AF}$ and $\mathrm{LV}$ low ejection fraction $^{9,10}$. The rapid increase of these long procedures has asked for a correct and optimal anaesthetic management in order to support and monitor patients correctly, help operator and reduce the duration of the procedure.

Nowadays, there aren't defined assessments, hence we had started this study to spread our single centre experience of HTEA and ablation catheter procedure (ACP). In specific type of ablations, the local anaesthesia and sedation could be more satisfactory in the management of some type of supraventricular tachyarrhythmia in quite, tolerate and still patients. On the other hand, some patients are unable to be firm, flat, or tolerate the procedure awake, children, adolescents, anxious adults, adults with congenital heart disease or complex cardiac anatomy, patients with respiratory disease and/or anticipated long and protracted procedures.

HTEA is largely employed to awake patients undergoing cardio-thoracic surgery and could provide an opportunity for patients with primary and secondary pulmonary impairments ${ }^{11-14}$. Indeed, the scientific literature and the clinical experience have already demonstrated the high risk of respiratory complications after general anaesthesia in patients with obesity, chronic obstructive disease, pulmonary fibrosis and so on ${ }^{12-15}$. The use of HTEA in AF ablation procedure as our protocol requires expertise of anesthesiological team, in particularly to reduce the risk Epidural Haematoma (EH) after heparin administration ${ }^{16}$. According to data of metaanalysis of Svircevic et $\mathrm{al}^{17}$, the risk of $\mathrm{EH}$ is about 1:1500 in cardiac surgery. Dajani et al ${ }^{18}$ documented a risk of 1:7500 every insertion in cardiac surgery since the 1999.

We highlight the HTEA requires normal coagulation pattern and the interruption of antiaggregation therapy such as clopidogrel and ticlopine, and during the procedure the operator must wait at least 60 minutes after epidural catheter placement to administer heparin.

The HTEA has many advantages like the optimal analgesia without respiratory depression (less control of respiratory gas exchanges) and the possibility of interaction with awake patient to monitor and check intraoperative complications immediately as stroke, transient 
ischemic attack and so on. The main cause of neurological complications could be the accumulation of char on the tip of the ablation electrode: we avoided that by irrigating catheters with saline/heparin 2-30 $\mathrm{ml} / \mathrm{min}$.

According to the literature and our experience, the other types of anaesthesia for ablation catheter procedure could be local anaesthesia for supraventricular tachyarrhythmia in order to prevent the suppression of the arrhythmia during the procedure, but the patient were able to be firm and immobile ${ }^{19}$. General anaesthesia (GA) was greater for the most of ACPs, but AV nodal conduction slowed with propofol and ventricular repolarisation was prolonged with isoflurane. Moreover, GA had the risks of propofol infusion syndrome and metabolic acidosis ${ }^{20}$.

\section{CONCLUSION}

HTEA could be available and reliable anesthetic management for high risk patient undergoing to endocardial AF ablation. This technique require a skilled and dedicated anesthesiological team and could help to have wakeful, comfortable and cooperative patient with a good intra operative pain control. These characteristics could improve the procedure in order of quality, timing and results. Even if HTEA needs more clinical experiences to confirm and define its role in $\mathrm{AF}$ ablation anesthesia.

All procedures followed were in accordance with the ethical standards of the responsible committee on human experimentation (institutional and national) and with the Helsinki Declaration of 1964 and later revisions.

\section{REFERENCES}

[1] Heeringa J, Conway DS, van der Kuip DA, et al. A longitudinal population-based study of prothrombotic factors in elderly subjects with atrial fibrillation: the Rotterdam Study 19901999. J Thromb Haemost. 2006. doi:10.1111 /j.1538-7836.2006.02115.x.

[2] Heeringa $J$ van der KDHAKJ van HGSB STLGWJ. Prevalence, incidence and lifetime risk of atrial fibrillation: the Rotterdam study. Eur Hear J. 2006. doi:10.1093/eurhear tj/ehi82510.1093/eurheartj/ehi651.

[3] C. M, F. DS, S. S, et al. Contribution of atrial fibrillation to incidence and outcome of ischemic stroke: Results from a populationbased study. Stroke. 2005.

[4] Komatsu T, Sato Y, Ozawa M, et al. Comparison between CHADS2 and CHA 2DS2- VASc score for risk stratification of ischemic stroke in Japanese patients with non- valvular paroxysmal atrial fibrillation not receiving anticoagulant therapy. Int Heart J. 2014. doi:10.1536/ihj.13-242.

[5] R. RYA, E. AA, S. RR, et al. CHA2DS2-VASc vs. CHADS2 at predicting the risk of stroke and death in a community-based cohort of patients with non-valvular atrial fibrillation who are on anticoagulation. Eur Heart J. 2014.

[6] M. H, M. H, O. R, Y. R, M. L. Risk prediction in atrial fibrillation. Comparison between the CHADS2, CHA2DS2-VASC score in a large contemporary cohort of patients with incident non-valvular atrial fibrillation. Europace. 2015.

[7] Haywood G. Can we ablate permanent atrial fibrillation? Heart. 2006. doi:10.1136/ hrt. 2005.075945.

[8] American Society of Regional Anesthesia and Pain Medicine (ASRA), Neal JM, Mulroy MF, Weinberg GL, American Society of Regional A, Pain M. American Society of Regional Anesthesia and Pain Medicine checklist for managing local anesthetic systemic toxicity: 2012 version. Reg Anesth Pain Med. 2012. doi:10.1097/AAP.0b013e31822e0d8a.

[9] Themistoclakis S, Tritto M, Bertaglia E, et al. [Catheter ablation of atrial fibrillation: Health Technology Assessment Report from the Italian Association of Arrhythmology and Cardiac Pacing (AIAC)]. G Ital Cardiol. 2011. doi:10.1714/966.10545.

[10] Earley MJ, Schilling RJ. Catheter and surgical ablation of atrial fibrillation. Heart. 2006; 92(2):266-274. doi:10.1136/ hrt.2005.06 7389.

[11] Hemmerling TM, Noiseux N, Basile F, Noël MF, Prieto I. Awake cardiac surgery using a novel anesthetic technique. Can J Anesth. 2005. doi:10.1007/BF03021610.

[12] S. C, G. L, F. M, et al. Aortic valve replacement on two octogenarians awake patients without tracheal intubation, with thoracic epidural anesthesia. G Ital Cardiol. 2006.

[13] Noiseux N, Prieto I, Bracco D, Basile F, Hemmerling T. Coronary artery bypass grafting in the awake patient combining high thoracic epidural and femoral nerve block: First series of 15 patients. Br J Anaesth. 2008. doi:10.1093 /bja/aem370.

[14] Kiss G, Claret A, Desbordes J, Porte H. Thoracic epidural anaesthesia for awake thoracic surgery in severely dyspnoeic patients excluded from general anaesthesia. Interact Cardiovasc Thorac Surg. 2014. doi:10.1093/ icvts/ivu230.

[15] G. K, O. J, S. H, R. A, J. D, E. R. Perioperative management of thoracic epidural anaesthesia for awake thoracic surgery in dyspneic patients excluded from general anaesthesia. Eur $J$ Anaesthesiol. 2012. 
[16] Cabrera A, Ornaque I, Obarrio LL, et al. [Epidural hematoma after catheter removal in a patient without coagulation disorders] TL - 49. [Epidural hematoma after Catheter Remov a patient without Coagul Disord. 2002.

[17] Svircevic V, van Dijk D, Nierich AP, et al. Meta-analysis of thoracic epidural anesthesia versus general anesthesia for cardiac surgery. Anesthesiology. 2011;114(2):271-282. doi:10. 1097/ ALN. 0b013e318201d300.

[18] Djaiani G, Fedorko L, Beattie WS. Regional anesthesia in cardiac surgery: a friend or a foe? Semin Cardiothorac Vasc Anesth. 2005.

[19] Boynes SG. Moore PA. Shepherd CJ. Bennett C. Anesthetic management. Oral Surg Oral Med Oral Pathol Oral Radiol Endod. 2005;99(2):E8-E10.

[20] Ashley EMC. Anaesthesia for electro physiology procedures in the cardiac catheter laboratory. Contin Educ Anaesthesia, Crit Care Pain. 2012; 12(5): 230- 236. doi: 10. 1093/bjaceaccp/mks03.

Citation: Angela Lappa, Marzia Cottini, Patrizia Picozzi, Silvia Donfrancesco, Davino Emilio, Valentina Coltelli et al. High Thoracic Epidural Anaesthesia for Endocardial Ablation of Atrial Fibrillation: A Single Centre Experience. ARC Journal of Anesthesiology. 2017;2(1):7-11. DOI: dx.doi.org/10.20431/24559792.0201003.

Copyright:(C) 2017 Authors. This is an open-access article distributed under the terms of the Creative Commons Attribution License, which permits unrestricted use, distribution, and reproduction in any medium, provided the original author and source are credited. 